\title{
Front Matter: Volume 7037
}

, "Front Matter: Volume 7037," Proc. SPIE 7037, Carbon Nanotubes and Associated Devices, 703701 (29 September 2008); doi: 10.1117/12.806037

SPIE Event: NanoScience + Engineering, 2008, San Diego, California, United States 


\title{
PROCEEDINGS OF SPIE
}

\section{Carbon Nanotubes and Associated Devices}

\author{
Manijeh Razeghi \\ Didier Pribat \\ Young Hee Lee \\ Editors
}

10-12 August 2008

San Diego, California, USA

Sponsored and Published by

SPIE

Volume 7037 
The papers included in this volume were part of the technical conference cited on the cover and title page. Papers were selected and subject to review by the editors and conference program committee. Some conference presentations may not be available for publication. The papers published in these proceedings reflect the work and thoughts of the authors and are published herein as submitted. The publisher is not responsible for the validity of the information or for any outcomes resulting from reliance thereon.

Please use the following format to cite material from this book:

Author(s), "Title of Paper," in Carbon Nanotubes and Associated Devices, edited by Manijeh Razeghi, Didier Pribat, Young Hee Lee, Proceedings of SPIE Vol. 7037 (SPIE, Bellingham, WA, 2008) Article CID Number.

ISSN 0277-786X

ISBN 9780819472571

Published by

SPIE

P.O. Box 10, Bellingham, Washington $98227-0010$ USA

Telephone +1 3606763290 (Pacific Time) · Fax +1 3606471445

SPIE.org

Copyright (c) 2008, Society of Photo-Optical Instrumentation Engineers

Copying of material in this book for internal or personal use, or for the internal or personal use of specific clients, beyond the fair use provisions granted by the U.S. Copyright Law is authorized by SPIE subject to payment of copying fees. The Transactional Reporting Service base fee for this volume is $\$ 18.00$ per article (or portion thereof), which should be paid directly to the Copyright Clearance Center (CCC), 222 Rosewood Drive, Danvers, MA 01923. Payment may also be made electronically through CCC Online at copyright.com. Other copying for republication, resale, advertising or promotion, or any form of systematic or multiple reproduction of any material in this book is prohibited except with permission in writing from the publisher. The CCC fee code is 0277-786X/08/\$18.00.

Printed in the United States of America.

Publication of record for individual papers is online in the SPIE Digital Library.

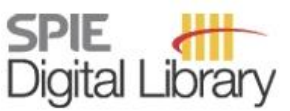

SPIEDigitalLibrary.org

Paper Numbering: Proceedings of SPIE follow an e-First publication model, with papers published first online and then in print and on CD-ROM. Papers are published as they are submitted and meet publication criteria. A unique, consistent, permanent citation identifier (CID) number is assigned to each article at the time of the first publication. Utilization of CIDs allows articles to be fully citable as soon they are published online, and connects the same identifier to all online, print, and electronic versions of the publication. SPIE uses a six-digit CID article numbering system in which:

- The first four digits correspond to the SPIE volume number.

- The last two digits indicate publication order within the volume using a Base 36 numbering system employing both numerals and letters. These two-number sets start with $00,01,02,03,04,05$, 06, 07, 08, 09, OA, OB ... 0Z, followed by 10-1Z, 20-2Z, etc.

The CID number appears on each page of the manuscript. The complete citation is used on the first page, and an abbreviated version on subsequent pages. Numbers in the index correspond to the last two digits of the six-digit CID number. 


\section{Contents}

vii Conference Committee

ix Revivals of molecular nonlinear optics in physics, chemistry, and life sciences (Plenary Paper) [7040-100]

J. Zyss, Lab. de Photonique Quantique et Moléculaire, CNRS, Institut d'Alembert (France)

\section{SESSION 1 DEVICES AND DEVICE PHYSICS I}

703702 High frequency carbon nanotube devices (Invited Paper) [7037-01]

M. F. Goffman, N. Chimot, E. Mile, M. C. Monteverde, J.-P. Bourgoin, V. Derycke, Lab. d'Electronique Moléculaire, DSM, IRAMIS, SPEC, CEA-Saclay (France)

703703 Electrical properties of carbon nanotube FETs (Invited Paper) [7037-02]

T. Mizutani, Y. Ohno, S. Kishimoto, Nagoya Univ. (Japan)

\section{SESSION 2 SYNTHESIS AND CHARACTERIZATION}

703706 Cross-polarized spectroscopy of DNA-wrapped nanotubes: unraveling the nature of optical response (Invited Paper) [7037-05]

S. V. Rotkin, Lehigh Univ. (United States) and Ctr. for Advanced Materials and Nanotechnology (United States); S. E. Snyder, Lehigh Univ. (United States)

703707 Resonant Raman scattering of smallest single-walled carbon nanotubes [7037-06] Z. K. Tang, Hong Kong Univ. of Science and Technology (Hong Kong China); J. P. Zhai, Hong Kong Univ. of Science and Technology (Hong Kong, China) and Shenzhen Univ. (China); Y. J. Feng, X. J. Hu, Hong Kong Univ. of Science and Technology (Hong Kong, China); R. Saito, Tohoku Univ. and CREST, JST (Japan); P. Sheng, Hong Kong Univ. of Science and Technology (Hong Kong, China)

703708 The application of dielectrophoresis on the characterization of electric property in multiwalled carbon nanotubes [7037-07]

C. Wei, C.-H. Liang, T.-Y. Wei, Tatung Univ. (Taiwan)

\section{SESSION 3 CNT FILMS AND INTERCONNECTS}

7037 OA Recent progress in carbon nanotube-based flexible transparent conducting film (Invited Paper) [7037-09]

H.-Z. Geng, K. K. Kim, Y. H. Lee, Sungkyunkwan Univ. (South Korea)

7037 OD An application of carbon nanotubes for integrated circuit interconnects [7037-12] J. C. Coiffic, CEA, Leti-Minatec (France); L. E. Foa Torres, CEA, Iniac (France); H. Le Poche, CEA, Liten (France); M. Fayolle, CEA, Leti-Minatec (France); S. Roche, CEA, Iniac (France); S. Maitrejean, CEA, Leti-Minatec (France); S. Roualdes, A. Ayral, IEM (France) 
7037 OE Electromechanical transducers based on single-walled carbon nanotubes (Invited Paper) [7037-13]

C. Stampfer, A. Jungen, T. Helbling, L. Durrer, C. Hierold, ETH Zurich (Switzerland)

7037 OF Carbon nanotube based microwave varactor: modeling, simulation, and design [7037-14] T. Ricart, S. Pacchini, LAAS-CNRS (France); C. S. Cojocaru, D. Pribat, LPICM, Ecole Polytechnique (France); D. Dubuc, LAAS-CNRS (France) and Univ. of Toulouse (France); K. Grenier, LAAS-CNRS (France)

\section{SESSION $5 \quad$ CNT INTERACTIONS WITH LIQUID CRYSTALS}

7037 Ol Electrokinetic elongation and subsequent dispersion of carbon nanotubes in liquid crystal medium (Invited Paper) [7037-17]

S. H. Lee, S. J. Jeong, A. K. Srivastava, M. Kim, E. M. Jo, Chonbuk National Univ. (South Korea); J. J. Bae, Y. H. Lee, Sungkyunkwan Univ. (South Korea)

7037 OL Sparse multiwall carbon nanotube electrodes arrays for liquid crystal photonic devices [7037-20]

T. D. Wilkinson, X. Wang, H. Butt, Ranjith R., W. I. Milne, Univ. of Cambridge (United Kingdom)

\section{SESSION 6 CNT ORGANIZATION AND SEPARATION}

7037 OM Patterning of single-walled carbon nanotubes using a low-fluence excimer laser photoablation process (Invited Paper) [7037-21]

J. Chae, X. Ho, J. A. Rogers, K. Jain, Univ. of Illinois at Urbana-Champaign (United States)

7037 ON Novel approach to align carbon nanotubes for planar type devices [7037-22]

D. Pribat, M. Gowtham, C. Cojocaru, B. Marquardt, A. Q. L. Quang, Lab. de Physique des Interfaces et des Couches Minces, Ecole Polytechnique (France); B. S. Kim, Samsung Electronics (South Korea)

703700 Light-induced accumulation of single-wall carbon nanotubes dispersed in aqueous solution [7037-23]

S. Shoji, T. Roders, Osaka Univ. (Japan); Z. Sekkat, Al Akhawayn Univ. (Morocco) and Academy Hassan II of Science and Technology (Morocco); S. Kawata, Osaka Univ. (Japan) and RIKEN (Japan)

\section{SESSION 7 FIELD EMISSION FROM CNTS}

7037 OP The application of carbon nanotube electron sources to the electron microscope (Invited Paper) [7037-25] M. Mann, Univ. of Cambridge (United Kingdom); M. El Gomati, T. Wells, The Univ. of York (United Kingdom); W. I. Milne, K. B. K. Teo, Univ. of Cambridge (United Kingdom) 
$7037 \mathrm{OQ}$ Surface plasmons localization on field emitters arrays for microwave modulation of tunneling currents [7037-26]

P. Guiset, S. Combrie, A. De Rossi, Thales Research \& Technology (France); M. Carras, Alcatel-Thales III-V Lab. (France); J. P. Schnell, P. Legagneux, Thales Research \& Technology (France)

7037 OR Field emission properties of multi-wall carbon nanotubes [7037-27]

Z.-C. Feng, Y.-Z. Huang, National Taiwan Univ. (Taiwan); J.-H. Ting, National Nano Device

Labs. (Taiwan); L.-C. Chen, National Taiwan Univ. (Taiwan); W. Lu, Fisk Univ. (United States)

\section{SESSION 8 DEVICES AND DEVICE PHYSICS II}

7037 OU Mass transport of lithium ions intercalated in pyrene-functionalized single-walled carbon nanotubes: a facile way to Schottky diode (Invited Paper) [7037-30]

H. C. Choi, H. Lim, Pohang Univ. of Science and Technology (South Korea)

7037 OW Synthesis of ZnO nanowires for thin film network transistors [7037-32]

S. H. Dalal, H. E. Unalan, Y. Zhang, P. Hiralal, L. Gangloff, A. J. Flewitt, G. A. J. Amaratunga, W. I. Milne, Univ. of Cambridge (United Kingdom)

7037 0X Atomic, electronic, and transport properties of quasi-one-dimensional nanostructures [7037-33]

Y. Xue, A. Hmiel, C. Stiles, SUNY, Univ. at Albany (United States)

\section{SESSION 9 SENSORS}

7037 OY Improving the performance of functionalized carbon nanotube thin film sensors by fluctuation enhanced sensing (Invited Paper) [7037-34]

A. Kukovecz, P. Heszler, Univ. of Szeged (Hungary); K. Kordás, Univ. of Oulu (Finland); S. Roth, Max Planck Institute for Solid State Research (Germany); Z. Kónya, H. Haspel, Univ. of Szeged (Hungary); R. Ionescu, Rovira i Virgili Univ. (Spain); A. Sápi, Univ. of Szeged (Hungary); J. Maklin, Univ. of Oulu (Finland); M. Mohl, Univ. of Szeged (Hungary) and Rice Univ. (United States); Z. Gingl, Univ. of Szeged (Hungary); R. Vajtai, Rice Univ. (United States) and Rensselaer Polytechnic Institute (United States); I. Kiricsi, Univ. of Szeged (Hungary); P. M. Ajayan, Rice Univ. (United States)

$7037 \mathrm{OZ}$ The use of nanotube structures in reducing the turn-on voltage in micro-discharges and micro-gas sensors (Invited Paper) [7037-35]

M. Tweedie, N. Soin, P. Kumari, S. S. Roy, A. Mathur, C. Mahony, P. Papakonstantinou, Univ. of Ulster (United Kingdom); J. A. D. McLaughlin, Univ. of Ulster (United Kingdom) and Sensor Technology and Devices Ltd. (United Kingdom)

703710 Fast fully plastic actuator based on ionic-liquid-based bucky gel [7037-36] K. Asaka, K. Mukai, I. Takeuchi, K. Kiyohara, T. Sugino, N. Terasawa, K. Hata, National Institute of Advanced Industrial Science and Technology (Japan); T. Fukushima, Advanced Science Institute, RIKEN (Japan); T. Aida, JST National Museum of Emerging Science and Innovation (Japan) 
703713 Carbon nanotubes and optical confinement: controlling light emission in nanophotonic devices (Invited Paper) [7037-40]

M. Steiner, F. Xia, IBM T. J. Watson Research Ctr. (United States); H. Qian, LudwigMaximilians-Univ. München (Germany); Y.-M. Lin, IBM Thomas J. Watson Research Ctr. (United States); A. Hartschuh, Ludwig-Maximilians-Univ. München (Germany); A. J. Meixner, Eberhard Karls Univ. Tübingen (Germany); P. Avouris, IBM Thomas J. Watson Research Ctr. (United States)

\section{POSTER SESSION}

703716 Carbon nanotube: polymer nanocomposites for possible pressure sensor applications [7037-37]

S. J. Varma, S. Jayalekshmi, Cochin Univ. of Science and Technology (India)

703717 Buckypaper from thin multiwalled carbon nanotubes [7037-43]

J. T. Han, H. J. Jeong, G.-W. Lee, Korea Electrotechnology Research Institute (South Korea)

703719 Micro-interconnection technology suitable for RF-NEMS varactors elaboration [7037-45] S. Pacchini, LAAS-CNRS (France) and Univ. of Toulouse (France); M. Dilhan, T. Ricart, LAASCNRS (France); C. Cojocaru, D. Pribat, LPICM (France); D. Dubuc, LAAS-CNRS (France) and Univ. of Toulouse (France); K. Grenier, LAAS-CNRS (France)

7037 1A Gigahertz resonance characteristics of nanotube linear motor [7037-46] J.-W. Kang, Y. G. Choi, G. H. Ryu, C. S. Won, Chungju National Univ. (South Korea)

7037 iB Raman nanometrology of graphene on arbitrary substrates and at variable temperature [7037-47]

I. Calizo, S. Ghosh, D. Teweldebrhan, W. Bao, F. Miao, C. N. Lau, A. A. Balandin, Univ. of California, Riverside (United States)

7037 1C Schematics and simulations for nanoscale engine based on nanotube encapsulating condensed gases [7037-48]

J.-W. Kang, C. S. Won, Y. G. Choi, Chungju National Univ. (South Korea)

7037 ID Characterization of nanotube nonvolatile memory device [7037-49]

J.-W. Kang, Chungju National Univ. (South Korea)

$7037 \mathrm{IE}$ Embedded multi-frequency generators based on multi-walled carbon nanotube [7037-50] J.-W. Kang, G. H. Ryu, C. S. Won, Y. G. Choi, Chungju National Univ. (South Korea)

$7037 \mathrm{lF}$ The study of physical properties and their effect on electro-optical characteristics of nematic liquid crystal doped with carbon nano tubes [7037-51]

A. K. Srivastava, M. Kim, E. Jo, H. Duschl, S. H. Lee, Chonbuk National Univ. (South Korea); H. K. Lee, S. E. Lee, Merck Advanced Technologies Ltd. (South Korea); J. J. Bae, Y. H. Lee, Sungkyunkwan Univ. (South Korea) 


\title{
Conference Committee
}

\author{
Symposium Chairs
}

David L. Andrews, University of East Anglia Norwich (United Kingdom) James G. Grote, Air Force Research Laboratory (United States)

Kevin J. Liddane, Oerlikon Optics USA Inc. (United States)

Conference Chairs

Manijeh Razeghi, Northwestern University (United States)

Didier Pribat, École Polytechnique (France)

Young Hee Lee, Sungkyunkwan University (South Korea)

Program Committee

Phaedon Avouris, IBM Thomas J. Watson Research Center (United States)

Ray H. Baughman, The University of Texas at Dallas (United States)

Jean-Philippe Bourgoin, CEA Saclay (France)

Hongjie Dai, Stanford University (United States)

Nicole Grobert, University of Oxford (United Kingdom)

Kenji Hata, National Institute of Advanced Industrial Science and Technology (Japan)

Ali Javey, University of California, Berkeley (United States)

S. H. Lee, Chonbuk National University (Korea, Republic of)

Pierre Legagneux, Thales Research \& Technology (France)

Annick Loiseau, ONERA (France)

Ryan P. McClintock, Northwestern University (United States)

William I. Milne, University of Cambridge (United Kingdom)

John A. Rogers, University of Illinois at Urbana-Champaign (United States)

Siegmar Roth, Max-Planck-Institut für Festkörperforschung (Germany)

Donald J. Silversmith, Air Force Office of Scientific Research (United States)

Jin Zhang, Peking University (China)

\section{Session Chairs}

1 Devices and Device Physics I

Young Hee Lee, Sungkyunkwan University (South Korea)

2 Synthesis and Characterization

Manish Chhowalla, Rutgers University (United States) 
3 CNT Films and Interconnects

Didier Pribat, Ecole Polytechnique (France)

4 NEMS

Takashi Mizutani, Nagoya University (Japan)

5 CNT Interactions with Liquid Crystals

Marcello F. Goffman, Commissariat à l'Energie Atomique (France)

6 CNT Organization and Separation

Slava V. Rotkin, Lehigh University (United States)

$7 \quad$ Field Emission from CNTs

Manijeh Razeghi, Northwestern University (United States)

8 Devices and Device Physics II

Mark Mann, University of Cambridge (United Kingdom)

9 Sensors

Kenji Hata, National Institute of Advanced Industrial Science and Technology (Japan)

10 Photonic and Photovoltaic Devices

Akos Kukovecz, University of Szeged (Hungary) 